\title{
FSRM Feedback Algorithm based on Learning Theory
}

\author{
Zhang Shui-Li*, Dong Jun-Tang and Gao Ying \\ College of Physics and Electronic Information, Yan'an University, Yan'an, Shaanxi Province, 716000, P.R. China
}

\begin{abstract}
In order to resolve the "semantic gap" problem between the image low-level features and high-level semantic features, this paper proposed a FSRM algorithm based on the learning theory. To compress the dimension of FSRM, the algorithm divided the image database into "related" and "irrelevant" two classes by retrieval of low-level features image. Then, the weights were adjusted in FSRM based on user feedback. Finally, after a finite time feedback, the weights were adjusted in FSRM using the learning theory FSRM algorithm, and the new retrieval results were returned to the user. The experiment shows that this algorithm can express the semantics contained in the image and can present a good description of the semantic similarity between images; therefore, the proposed algorithm has certain significance.
\end{abstract}

Keywords: FSRM, image retrieval, relevance feedback, semantic gap, semantic similarity.

\section{INTRODUCTION}

With the development of multimedia technology and internet, a vast multimedia information ocean appeared in front of people that further led to the super large image information base. The problem, how to effectively help people to find the needed information quickly and accurately, has become the core problem to be solved in image retrieval system [1]. The low-level features such as color and texture do not always accurately reflect the human visual perception of the high-level concept in CBIR. Feedback technology, which exactly solves the "semantic gap" problem underlying semantic features between images, has attracted the attention of many scholars. So far, the relevance feedback technology in the CBIR is broadly divided into two categories $[2,3]$ : One is adjusting some of the parameters in similarity measure based on the user's feedback information; the other is from a probability standpoint, calculating the probability of each image in the image library which meets user requirements, and taking the high probability images as the search results returned to the user. Cox [4] and Nuno [5] assumed that positive, negative feedback image is a series of independent retrieval unit for a given query image, and tried to minimize the retrieval error by using Bayes rule to optimize the search results. Rui and Huang proposed a feature standard deviation algorithm [6,7]. Their idea is reducing the weight of the larger feature dimension of the standard deviation in the feature space, and vice versa is increasing the weight. At the same time, they proposed a multilevel image model, and dealt with the problem of adjusting the weights by using global optimization methods based on the model. This paper presents a feedback algorithm based on fuzzy semantic relevant matrix (FSRM). The specific approach is: Firstly, divide the image library into relevant and irrelevant

*Address correspondence to this author at the College of Physics and Electronic Information, Yan'an University, Yan'an, Shaanxi Province, 716000, P.R. China; Tel: 18509110138,18329918059; Fax: 0911-2332045;

E-mail: zhangshuili74@163.com image categories by using low-level visual features; Secondly, adjust the weights in the FSRM in accordance with the relevant or irrelevant images user labeled; Finally, after a limited feedback times, further amendment of FSRM matrix elements should be conducted through learning theory, to better achieve the retrieval results. The proposed algorithm in this paper is feedback based on FSRM, so no prior knowledge of specific issues is needed. Moreover, the algorithm is convenient to implement and can achieve better search effect under the condition of limited feedback times.

\section{FSRM FEEDBACK ALGORITHM}

\subsection{FSRM Theory}

In 1965, the theory of fuzzy mathematics, presented by Zadeh, was used to study the problem of fuzzy uncertainty, which expanded the characteristic value scope of the set $\{0,1\}$ to the interval $[0,1]$ of continuous values. So the value in the interval $[0,1]$ can be used to represent a certain extent to which an object can meet some concept. The closer the object corresponding value to 1 , the greater the degree of compliance with the concept; on the contrary, the smaller the degree of compliance.

The Fuzzy Matrix A is defined as [2, 8]:

$$
\mathrm{A}=\left(\mathrm{a}_{\mathrm{ij}}\right)_{\mathrm{m} \times \mathrm{n}} \text {, and } \mathrm{a}_{\mathrm{ij}} \in[0,1](\mathrm{i}=1,2, \ldots \mathrm{m} ; \mathrm{j}=1,2, \ldots, \mathrm{n})
$$

Because of the ambiguity of human language and semantics, it is very difficult to completely describe the image with precise or literal semantics, using the fuzzy semantic relevance matrix (FSRM) to build the semantic bridge [3]. The FSRM elements satisfy the equation (1):

$$
\begin{aligned}
& R(i, j) \in[0,1], R(i, j)=1, \quad \text { and } \\
& R(i, j)=R(j, i), i, j=0,1, \ldots, N-1
\end{aligned}
$$

where $\mathrm{N}$ is the number of images in the Image Library. $\mathrm{R}(\mathrm{i}, \mathrm{j})$, the direct similarity, reflects the similarity between two images $i$ and $j$. FRSM is a fuzzy similar matrix according to the definition of fuzzy matrix. 
The size of FSRM is decided by the number of images in the image library, thus the FSRM is a very large matrix for a large image library, and so the corresponding computation is enormous. In order to reduce the size of the FSRM, this paper firstly classifies image library into two classes of relevant and irrelevant, and then a FSRM is established for each class, thus the size of FSRM is greatly reduced.

\subsection{FSRM Feedback Algorithm Description}

For each class of image library, $R(i, j)$, the similarity of image $i$ and $j$, is represented by the element of FSRM, and FSRM is initialized to a symmetric matrix:

$$
\begin{aligned}
& 0 \leq R(i, j) \leq 1(i, j=1,2, \ldots, N) \text { if } \quad i=j \quad \text { then }: \quad R(i, j)=1 \\
& \text { otherwise } \quad R(i, j)=R(j, i)(i, j=1,2, \ldots, N)
\end{aligned}
$$

where $\mathrm{N}$ is the number of each image library. Because there are some similarities between the images for each image category, the initial value of FSRM is set to the vaguest value of 0.5 between $0-1$, that is $R(i, j)=0.5(i \neq j$ and $i, j=1,2, \cdots$, $\mathrm{N})$. During FSRM feedback process, for the relevant image class $\left(\mathrm{I}^{+}\right)$in the same image library, the corresponding weights in FSRM are adjusted according to the equation (3), or do not modify; unrelated image class $\left(\mathrm{I}^{-}\right)$in the same image library and the corresponding weights in FSRM are adjusted according to the equation (4).

$$
\begin{aligned}
& R(i, j)_{\text {new }}=R(i, j)_{\text {old }}+\alpha\left(1-R(i, j)_{\text {old }}\right) \\
& R(i, j)_{\text {new }}=R(i, j)_{\text {old }}-\beta\left(1-R(i, j)_{\text {old }}\right)
\end{aligned}
$$

where, $\alpha, \beta$ respectively express the degree parameter weights increased or decreased, and satisfy $\alpha+\beta=1 \quad(\alpha=0.35$ and $\beta=0.65$ ).

With FSRM weight adjustment equations, the experimental procedures can be obtained using FSRM for retrieval, and the specific steps are:

\section{(1) Initialize the FSRM.}

(2) After research based on low-level visual features, the former $\mathrm{K}(\mathrm{K}<\mathrm{N})$ images are chosen as the search results returned to the user, the users labeling the $\mathrm{k}$ images as the current relevant image database $\mathrm{I}_{1}^{+}$, the remainder being irrelevant image database $\mathrm{I}^{-}{ }_{1}$.
(3) According to the equation (3) and (4), each class of the image library weights respectively is adjusted in its FSRM.

(4) Partial weights of each image library in FSRM have been prepared from an initial value of 0.5 to a more realistic semantic value after training through a limited times of step 3, that is to say, a certain similarity has been established between images in each image class, then the semantic matrix is modified by learning each of the FSRM data according to the 2.3 section algorithm, and then the results are returned back to the user.

(5) According to the user feedback, the corresponding image in FSRM is marked with the weights in an order ranging from big to small, and the most similar images are returned to the user. If the user is satisfied, then the results are saved, terminating the retrieval. Otherwise, the second step is repeated again until the feedback results meet the user needs.

\subsection{Learning FSRM Feedback Algorithm}

There is a certain gap between similarity of low visual features and semantic similarity in image retrieval, therefore, the memory and learning of semantic information play an important role to improve the performance of retrieval system. Fig. (1) shows the top 10 fuzzy semantic correlations between the image and the query image in a FSRM after several feedbacks by using the algorithm of section 2.2 (weights matrix elements are rounded to retain two decimal places). As can be seen from Fig. (1), after a finite number of feedback times, some weights of each fuzzy semantic relevant matrix have been from the initial value $(0.5)$ to the semantic weights.

The elements in Fig. (1) represent the direct similarity between respective images. As can be seen, after several feedback times, $\mathrm{R}(7,4)$ is updated to 0.68 from the initial value of $0.5, R(3,6)$ is updated to 0.91 , but $R(5,3)$ is still the initial value 0.5 having no change due to the limitation of the feedback frequency, thus indicating that it is difficult to reflect their semantic similarity implied for the "direct similarity" $\mathrm{R}(5,3)$. In comparison, $\mathrm{R}(5,1) * \mathrm{R}(1,3)=0.6794$ can better reflect the hidden semantic information between image 5 and image 3 . It is necessary for the hidden semantic information embodied in FSRM for better research effect. The semantic similarity is defined as equation (5):

\begin{tabular}{|c|c|c|c|c|c|c|c|c|c|c|}
\hline & 1 & 2 & 3 & 4 & 5 & 6 & 7 & 8 & 9 & 10 \\
\hline 1 & 1 & 0.5 & 0.79 & 0.5 & 0.86 & 0.5 & 0.5 & 0.91 & 0.5 & 0 \\
\hline 2 & 0.5 & 1 & 0.5 & 0.5 & 0.5 & 0.5 & 0.91 & 0.5 & 0.5 & 0.5 \\
\hline 3 & 0.79 & 0.5 & 1 & 0.68 & 0.5 & 0.91 & 0.5 & 0.5 & 0.5 & 0.86 \\
\hline 4 & 0.5 & 0.5 & 0.68 & 1 & 0.5 & 0.5 & 0.68 & 0.5 & 0.5 & 0.5 \\
\hline 5 & 0.86 & 0.5 & 0.59 & 0.5 & 1 & 0.5 & 0.18 & 0.5 & 0.18 & 0.5 \\
\hline 6 & 0.5 & 0.5 & 0.91 & 0.5 & 0.5 & 1 & 0.5 & 0.46 & 0.5 & 0.5 \\
\hline 7 & 0.5 & 0.91 & 0.5 & 0.68 & 0.18 & 0.5 & 1 & 0.5 & 0.86 & 0.5 \\
\hline 8 & 0.91 & 0.5 & 0.5 & 0.5 & 0.5 & 0.46 & 0.5 & 1 & 0.5 & 0.5 \\
\hline 9 & 0.5 & 0.5 & 0.5 & 0.5 & 0.18 & 0.5 & 0.86 & 0.5 & 1 & 0.5 \\
\hline 10 & 0.5 & 0.5 & 0.86 & 0.5 & 0.5 & 0.5 & 0.5 & 0.5 & 0.5 & 1 \\
\hline
\end{tabular}

Fig. (1). FSRM matrix before learning. 


\begin{tabular}{|c|c|c|c|c|c|c|c|c|c|c|}
\hline & 1 & 2 & 3 & 4 & 5 & 6 & 7 & 8 & 9 & 10 \\
\hline 1 & 1 & 0.5 & 0.79 & 0.5 & 0.86 & 0.5 & 0.5 & 0.91 & 0.5 & 0.5 \\
\hline 2 & 0.5 & 1 & 0.5 & 0.5 & 0.5 & 0.5 & 0.91 & 0.5 & 0.5 & 0.5 \\
\hline 3 & 0.79 & 0.5 & 1 & 0.68 & 0.6794 & 0.91 & 0.5 & 0.5 & 0.5 & 0.86 \\
\hline 4 & 0.5 & 0.5 & 0.68 & 1 & 0.5 & 0.5 & 0.68 & 0.5 & 0.5 & 0.5 \\
\hline 5 & 0.86 & 0.5 & 0.6794 & 0.5 & 1 & 0.5 & 0.18 & 0.7826 & 0.18 & 0.5 \\
\hline 6 & 0.5 & 0.5 & 0.91 & 0.5 & 0.5 & 1 & 0.5 & 0.46 & 0.5 & 0.5 \\
\hline 7 & 0.5 & 0.91 & 0.5 & 0.68 & 0.18 & 0.5 & 1 & 0.5 & 0.86 & 0.5 \\
\hline 8 & 0.91 & 0.5 & 0.5 & 0.5 & 0.7826 & 0.46 & 0.5 & 1 & 0.5 & 0.5 \\
\hline 9 & 0.5 & 0.5 & 0.7189 & 0.5 & 0.18 & 0.5 & 0.86 & 0.5 & 1 & 0.5 \\
\hline 10 & 0.5 & 0.5 & 0.86 & 0.5 & 0.5 & 0.5 & 0.5 & 0.5 & 0.5 & 1 \\
\hline
\end{tabular}

Fig. (2). FSRM matrix after learning.

$$
\begin{array}{ll}
\text { if } & R(i, k) \geq T, R(k, j) \geq T, R(i, k) * R(k, j) \geq T \\
\text { and } & R(i, k) * R(k, j) \geq R(i, j) \geq 0.5, \\
\text { then } & R(i, j)=R(i, k) * R(k, j) \quad(i \neq j)
\end{array}
$$

where, $\mathrm{T}$ is the threshold value, and is taken 0.5 in the experiment.

Through the study of the above methods, the weights in Fig. (1) are adjusted to the results in Fig. (2) (element weights rounded to retain four decimal places). As can be seen from Fig. (2), after limited feedback learning, element in FSRM updates faster, and as a result, is closer to the reality of semantic similarity, thereby further improving the retrieval performance of the system.

\section{EXPERIMENTAL RESULTS AND ANALYSIS}

\subsection{Experimental Environment}

The simulation experiment is based on MATLAB platform; image library is composed of two types of images: character images (50 images) and flower images (50 images). Image size is $480 \times 640$ in each image database, image format is JPG. In experiment, around five individuals were randomly selected from colleagues for each class image library to retrieve two times. The user marked the result image as "relevant" or "irrelevant" class according to his requirements in each feedback.

\subsection{Experimental Results Analysis}

Experimental analysis consists of two parts: One part is effectiveness comparison of the three algorithms based on low level visual features (selected texture feature in the experiment), no relevant feedback learning and relevant feedback learning; another part is studying effectiveness of the proposed algorithm from the perspective of the number of feedbacks. Figs. (3-5) show the 8 most similar images for the retrieval of flower database. Fig. (3) gives the retrieval results based on low level visual features (texture features); Fig. (4) provides the retrieval results based on the no relevance feedback learning, only adjusting the weight in FSRM according to the user' $\mathrm{S}$ feedback information; Fig. (5) shows the results based on learning algorithm after a limited feedback time using no relevance feedback learning algorithm. The learning algorithm can effectively improve the performance of the system by comparison of Figs. (4-7) providing relationship curve of the average feedback precision and the number of feedbacks. Feedback precision is defined as equation (6):

$$
P=a / b
$$

where, $\mathrm{a}$ is the number of relevant image ranked in the top $\mathrm{K}$, $\mathrm{b}$ is the number of image ranked in the top K.

As Figs. (6 and 7) illustrate, the algorithm of learning FSRM can further improve the effectiveness of the retrieval system.

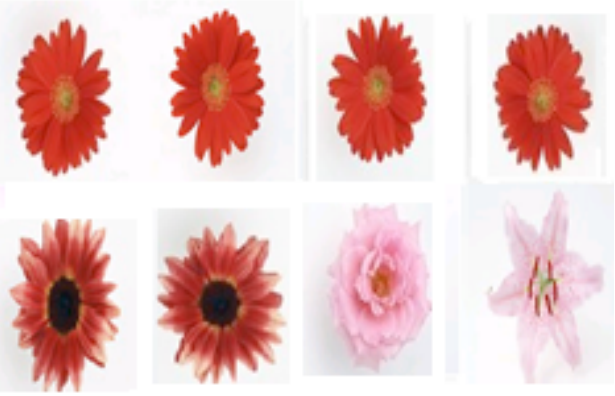

Fig. (3). Retrieval results based on low level visual features.

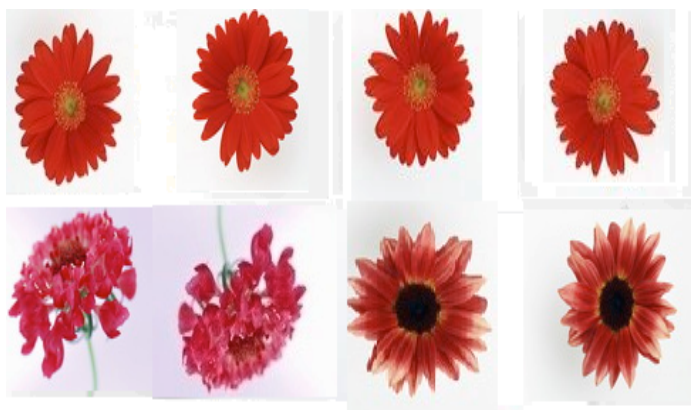

Fig. (4). First retrieval results before learning.

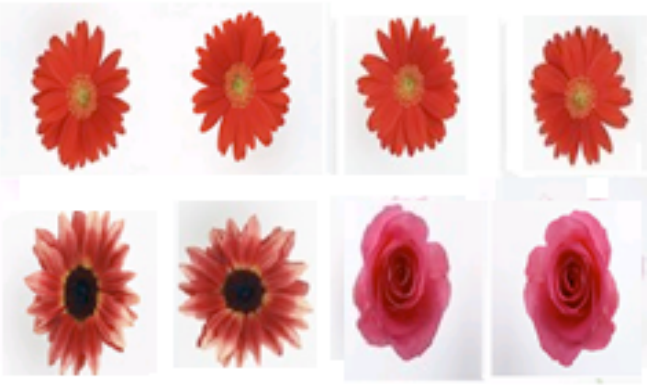

Fig. (5). First retrieval results after learning. 


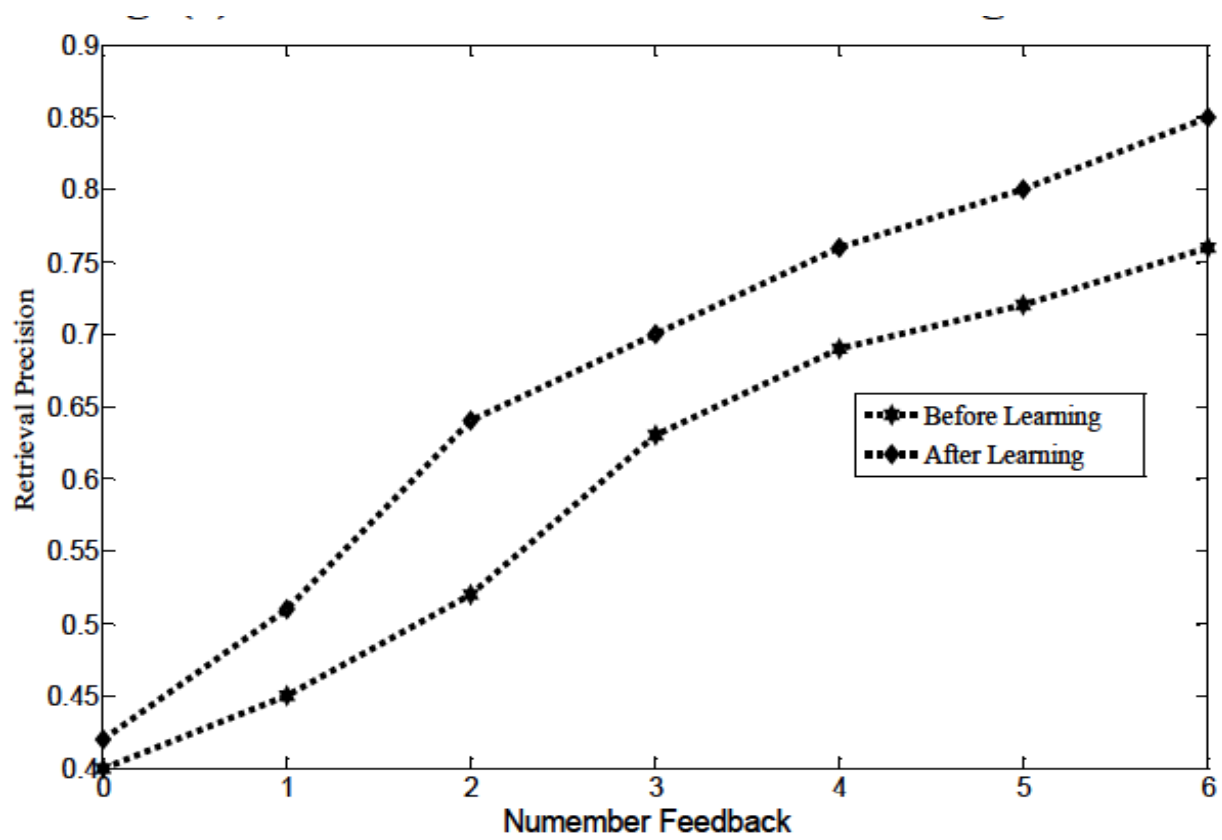

Fig. (6). Feedback precision of character.

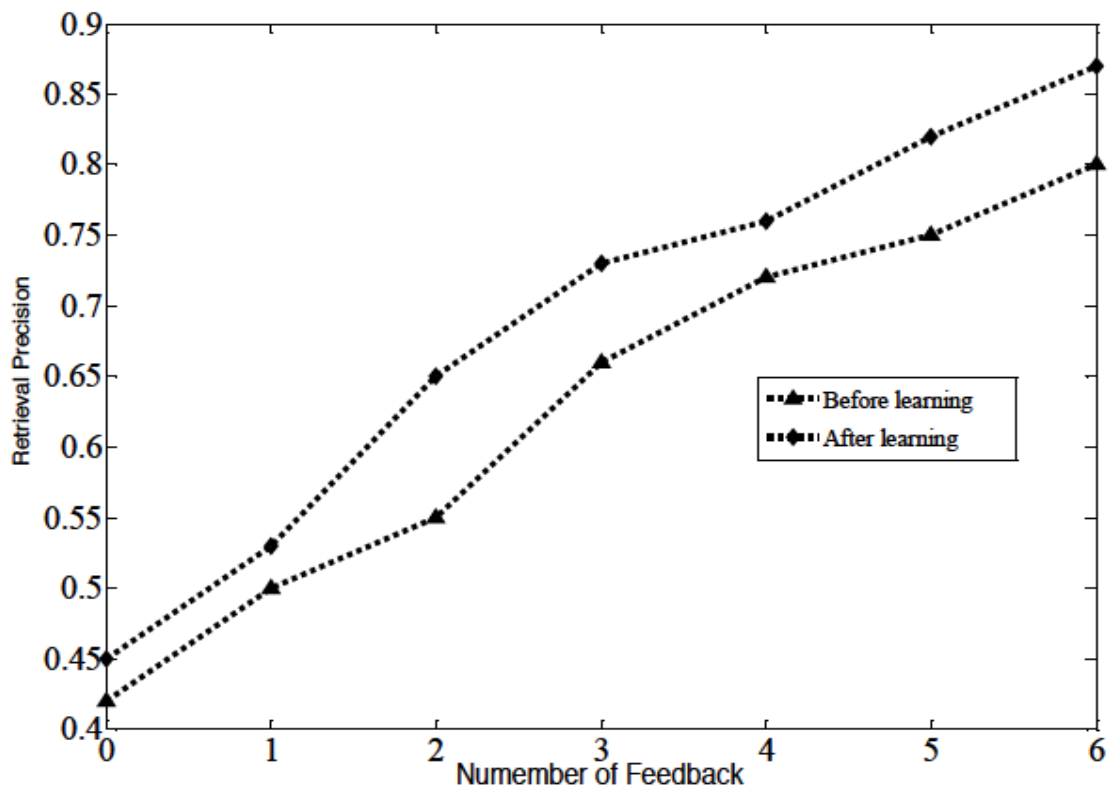

Fig. (7). Feedback precision of flower.

\section{CONCLUSION}

Relevance feedback plays an important role in image retrieval; therefore this paper applied the fuzzy semantic relevant matrix to relevance feedback of image retrieval, and proposed a FSRM feedback algorithm based on the learning theory. Firstly, the algorithm classified image library into two classes of relevant and irrelevant by using low level visual features to retrieval, and then a FSRM was established for each class, thus the size of FSRM was greatly reduced; Again, the weights were constantly adjusted in FSRM for each class according to the user' $\mathrm{s}$ feedback information; Finally, the weights were further updated in FSRM based on the learning proposed in section 2.3 after a limited feedback time using no relevance feedback learning algorithm, thus the weights were closer to the semantic features. Exper- imental results show that the proposed algorithm solves the "semantic gap" problem between low-level features and high-level features to a certain extent, and has certain application value.

\section{CONFLICT OF INTEREST}

The authors confirm that this article content has no conflict of interest.

\section{ACKNOWLEDGEMENTS}

This work was financially supported by the Shaanxi Natural Science Foundation (2014JM8357) and Yan'an University Youth Foundation (YDQ2014-37) 


\section{REFERENCES}

[1] X. Y. Tan, Z. X. Sun, and Z. Y. Fu, "Relevance feedback in content-based image retrieval: the state of the art", Journal of Nanjing University (Natural Sciences), vol. 40, pp. 639-648, 2004.

[2] S. H. Guo, and M. Yang, "Relevance feedback algorithm based on fuzzy semantic relevance matrix in image retrieval", Computer Science, vol. 39, pp. 540-542, 2012.

[3] H. J. Jin, Research on Image Retrieval Based on Semantic Feature, Harbin Institute of Technology China, 2006.

[4] I. J. Cox, T. P. Minka, T. V. Papathomas, and M.L. Miller, "The Bayesian image retrieval system, PicHunter: theory, implementation, and psychophysical experiments", IEEE Transactions on Image Processing, vol. 9, no. 1, pp. 20-37, 2000.
[5] N. Vasconcelos, and A. Lippman, "Learning from user feedback in image retrieval systems", Computer Vision - ECCV 2000, Lecture Notes in Computer Science, vol. 1842, pp. 33-47, 2000.

[6] Y. Rui, and T.S. Huang, "Relevance feedback: a power tool for interactive content-based image retrieval", IEEE Circuits and Systems for Video Technology, vol. 8, no. 5, pp. 644-655, 1999.

[7] Y. Rui, and T.S. Huang, "A novel relevance feedback technique in image retrieval", In: Buford, J., Ed. Proceedings of the $7^{\text {th }}$ ACM International Conference (part 2) on Multimedia (Part 2). New York, NY: ACM Press, 1999, pp. 67-70.

[8] Z. Peng, and Y. Sun, Fuzzy Mathematics and its Application, Wuhan University Press, China, pp. 75-80, 2003.

(C) Shui-Li et al.; Licensee Bentham Open.

This is an open access article licensed under the terms of the Creative Commons Attribution Non-Commercial License (http://creativecommons.org/licenses/by-nc/4.0/) which permits unrestricted, non-commercial use, distribution and reproduction in any medium, provided the work is properly cited. 